Fr equency domai $n$ detect $i$ on of coher ence mul ti pl exed sensor si gnal s by usi ng an optical I oop wi th a frequency shifter

\begin{tabular}{|l|l|}
\hline 著者 & I i yama Koi chi, Hayashi Ken- i chi , 飯山 宏一 \\
\hline $\begin{array}{l}\text { j our nal or } \\
\text { publ i cat i on t i t l e }\end{array}$ & J our nal of Li ght wave Technol ogy \\
\hline vol une & 15 \\
\hline number & 11 \\
\hline page r ange & $2069-2075$ \\
\hline year & $1997-11$ - 01 \\
\hline URL & ht t p: //hdl . handl e. net /2297/1785 \\
\hline
\end{tabular}




\title{
Frequency Domain Detection of Coherence Multiplexed Sensor Signals by Using an Optical Loop with a Frequency Shifter
}

\author{
Koichi Iiyama, Member, IEEE, and Ken-ichi Hayashi, Member, IEEE
}

\begin{abstract}
We describe a frequency domain detection scheme of coherence multiplexed sensor signals by using an optical loop with a frequency shifter. The optical path differences of the sensors are adjusted to integer-multiple of the loop length of the optical loop, and then the the sensor signals are multiplexed in the frequency domain, appearing at the integer-multiple of the working frequency of the frequency shifter. In the experiment, four Michelson-type sensors are successfully multiplexed with the sensitivities of about $2 \mathrm{mrad} / \sqrt{\mathrm{Hz}}$. The sensitivities are also theoretically calculated which are in good agreement with the experimental results. The optimum design of the optical loop is also discussed.
\end{abstract}

Index Terms - Erbium-doped fiber amplifier, multiplexing, optical fiber sensors, interferometers.

\section{INTRODUCTION}

$\mathbf{M}$ ULTIPLEXING fiber-optic sensors is an attractive technology because it will save cost due to reduction in the number of light sources, photodetectors and fiber transmission lines. Three different types of the multiplexing scheme have been investigated, which are the time division multiplexing [1]-[2], the frequency division multiplexing [3]-[5], and the coherence multiplexing [6]-[8]. The coherence multiplexed sensor system is composed of a light source, a set of sensing interferometers whose optical path differences (OPD's) are different each other, and a corresponding set of receiving interferometers. The OPD of each receiving interferometer must match to that of one of the sensing interferometers. The sensing and the receiving interferometers are arranged in tandem and are illuminated by a CW light source whose coherence length is much shorter than any OPD of the interferometers. The sensor signal imprinted on the light in one of the sensing interferometers can be retrieved by only the corresponding receiving interferometer due to the low coherence nature of the light source. In this system, $2 n$ interferometers ( $n$ sensing and $n$ receiving interferometers) and $n$ photodetectors are required for multiplexing $n$ sensors, and consequently the optical system becomes considerably large.

We have proposed a detection scheme of coherence multiplexed sensor signals by using an optical loop with a frequency

Manuscript received March 11, 1997.

$\mathrm{K}$. Iiyama is with the Department of Electrical and Computer Engineering, Faculty of Engineering, Kanazawa University, Kanazawa 920 Japan.

K. Hayashi, deceased, was with the Department of Electrical and Computer Engineering, Faculty of Engineering, Kanazawa University, Kanazawa 920 Japan.

Publisher Item Identifier S 0733-8724(97)08280-7.

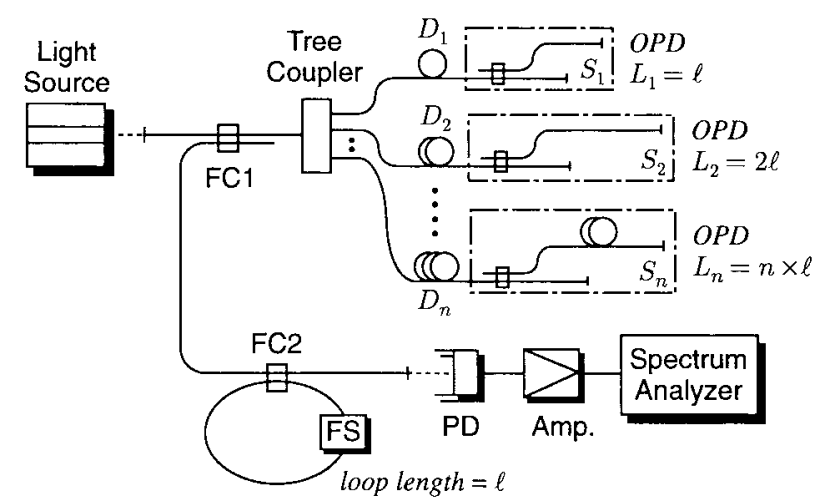

Fig. 1. Configuration of the proposed coherence multiplexed sensorsystem.

shifter [9], [10]. In the system, the OPD's of sensing interferometers are adjusted to integer-multiple of the loop length of the optical loop. The sensor signals are multiplexed in the frequency domain, appearing at the integer-multiple of the working frequency of the frequency shifter in the optical loop. The sensitivities of the sensors and the number of sensors to be multiplexed are determined by loop loss of the optical loop, and the loop loss must be reduced to enhance the sensitivities of the sensors and to increase the number of sensors to be multiplexed.

In this paper, we demonstrate the experimental results and the theoretical study of this system. In Section II, we describe the principle of this sensor system, and the crosstalk between sensors is also considered. In Section III, the experimental results are shown. In the experiment, an erbium-doped optical fiber amplifier was introduced into the optical loop to reduce the loop loss and to enhance the sensitivities of the sensors. The theoretical consideration of the sensitivity is discussed in Section IV.

\section{Configuration}

\section{A. Principle}

The configuration of the proposed system is shown in Fig. 1, in which $n$ Michelson-type interferometric sensors are arranged in parallel. This system consists of a low coherence light source, $n$ sensing interferometers $S_{k}\left(\mathrm{OPD}=L_{k}\right.$, $k=1,2, \cdots, n)$ and an optical loop with an optical frequency shifter (FS) working at $f_{F S} \mathrm{~Hz}$. The loop length of the optical loop is denoted by $\ell$. It is essential that the OPD $L_{k}$ is chosen 
as $L_{k}=k \times \ell$ and the loop length $\ell$ is chosen to be much longer than the coherence length of the light source.

The principle of this system is as follows. If we consider the interferometer $S_{1}$ and the optical loop together, the beam passing through a long path of $S_{1}$ and recirculating $i$-times in the loop can interfere with the beam passing through a short path of $S_{1}$ and recirculating $(i+1)$-times in the loop. Since the latter beam is more frequency-shifted by $f_{F S} \mathrm{~Hz}$ than the former, the interference signal, that is, the sensor signal appears around $f_{F S} \mathrm{~Hz}$. Similarly the sensor signal of $S_{k}$ appears around $\left(k \times f_{F S}\right) \mathrm{Hz}$. Therefore, all sensor signals of the sensor array are multiplexed in the frequency domain and can be detected separately by using electric bandpass filters. In this method, $n$ sensing interferometers and only a set of the optical loop and a photodetector are required for the $n$-sensor array. The numbers of the interferometers and the photodetectors are considerably reduced as compared with the conventional coherence multiplexed system where $2 n$ interferometers and $n$ photodetectors are required.

\section{B. Crosstalk}

In our sensor system shown in Fig. 1, if the beam passing through a long path of $S_{1}$ interferes with the beam passing through a short path of $S_{2}$ and recirculating $k$-times in the loop, the interference signal appears around $\left(k \times f_{F S}\right) \mathrm{Hz}$ in the frequency domain, which is the same frequency of the sensor signal of $S_{k}$. This interference is undesirable and is called crosstalk. The crosstalk degrades performance of the sensor system and must be avoided. In order to avoid the crosstalk, we insert delay lines with length $D_{k}(k=1,2, \cdots, n)$ in front of every sensing interferometer. They are long enough to move any crosstalk toward the higher frequency range than the intended signal frequency range. The length of the delay line $D_{k}$ is determined as follows. The crosstalk appeared at the lowest frequency is caused by the interference between the beam passing through a long path of $S_{k-1}$ and the beam passing through a short path of $S_{k}$. To avoid the crosstalk, the OPD between the above two beams must be longer than $(n+1) \ell$, where $n$ and $\ell$ are again the number of sensors to be multiplexed and the loop length, respectively. This is written as

$$
\begin{aligned}
& \left(2 D_{k}+L_{k}^{(S)}\right)-\left(2 D_{k-1}+L_{k-1}^{(L)}\right) \geq(n+1) \ell \\
& (k=2,3, \cdots, n)
\end{aligned}
$$

where $L_{k}^{(S)}$ is the length of the short path of $S_{k}$, and $L_{k-1}^{(L)}$ is the length of the long path of $S_{k-1}$. The factor of 2 indicates round-trip propagation. If we suppose that $L_{k}^{(S)}=L_{k-1}^{(S)}$, (1) can be written as

$$
D_{k} \geq \frac{n+k}{2} \ell+D_{k-1}
$$

Equation (2) is the condition for determining the length of delay line to avoid the crosstalk. For example, if $n=4$, the length of the delay lines $D_{2}, D_{3}$, and $D_{4}$ are determined as

$$
\left.\begin{array}{l}
D_{2} \geq 3 \ell+D_{1} \\
D_{3} \geq \frac{7}{2} \ell+D_{2} \\
D_{4} \geq 4 \ell+D_{3}
\end{array}\right\} .
$$

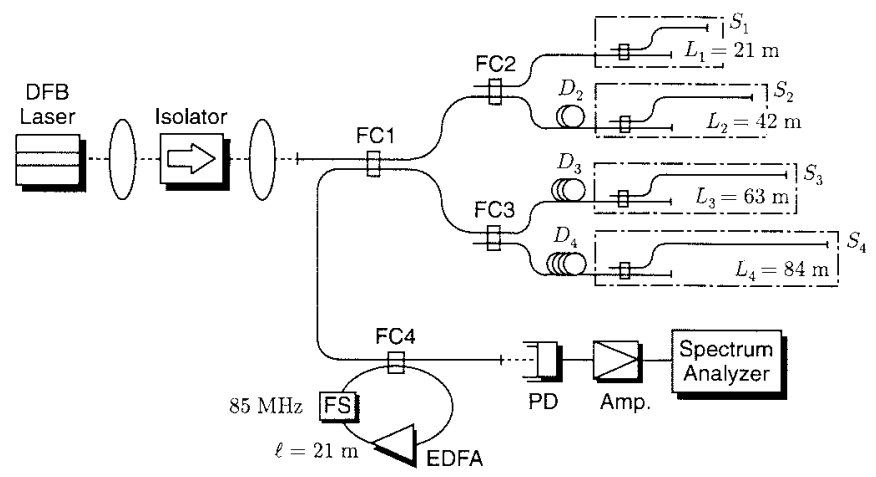

Fig. 2. Experimental setup.

\section{EXPERIMENTS}

The experimental setup is shown in Fig. 2, in which four Michelson-type sensing interferometers are multiplexed in parallel. In our experiments, the light source used was a DFB laser emitting at $1.53 \mu \mathrm{m}$ (Hitachi HL-1541), whose coherence length was about $15 \mathrm{~m}$. In this paper, the coherence length is defined as the OPD of a two-beam interferometer where insignificant interference occurs. An acousto-optic modulator (AOM: HOYA AF-150) was used as the frequency shifter, which was driven at $85 \mathrm{MHz}$. The insertion loss of the AOM was $8 \mathrm{~dB}$, and the total loop loss including losses at connectors and fusion splices was $8.7 \mathrm{~dB}$. The excess loss and the coupling coefficient of the fiber coupler (FC4) in the optical loop were $1 \mathrm{~dB}$ and $50 \%$, respectively. The detected signal was analyzed by a spectrum analyzer.

An erbium doped optical fiber amplifier (EDFA) was introduced into the optical loop to reduce the loop loss. As is shown in the next section, the sensitivity and the number of sensors to be multiplexed are dominated by the loop loss. In fact, when the EDFA was not introduced into the optical loop, we were able to multiplex only two sensors $\left(S_{1}\right.$ and $\left.S_{2}\right)$, and their sensitivities were about $0.8 \mathrm{mrad} / \sqrt{\mathrm{Hz}}$. The $\mathrm{Er}^{3+}$ concentration of the EDFA was $500 \mathrm{ppm}$, and Al was codoped. The EDFA was pumped by a $0.98 \mu \mathrm{m}$ semiconductor laser. The EDFA was $16 \mathrm{~m}$ long, and the resultant loop length of the optical loop $\ell$ was $\ell=21 \mathrm{~m}$. The OPD's of $S_{1}, S_{2}, S_{3}$, and $S_{4}$ were adjusted as $L_{1}=21 \mathrm{~m}, L_{2}=42 \mathrm{~m}, L_{3}=63 \mathrm{~m}$ and $L_{4}=84 \mathrm{~m}$, respectively. The optical power at the input port of the optical loop was about $10 \mu \mathrm{W}$. In this condition, the optical loop lased around $1.55 \mu \mathrm{m}$ when the pump power into the EDFA was above $1.5 \mathrm{~mW}$, and therefore, the pump power was adjusted to below $1.5 \mathrm{~mW}$ throughout the experiment.

Fig. 3 shows an example of the measured multiplexed sensor signal spectrum. The pump power to the EDFA was $1.5 \mathrm{~mW}$, and the resolution of the spectrum analyzer was $100 \mathrm{kHz}$. Four independent peaks are clearly observed in the spectrum. Their frequencies are $85 \mathrm{MHz}, 170 \mathrm{MHz}, 255 \mathrm{MHz}$ and $340 \mathrm{MHz}$, corresponding to the sensor signals of $S_{1}$, $S_{2}, S_{3}$, and $S_{4}$, respectively. The increases of noise level around $0 \mathrm{~Hz}, 85,170,255$, and $340 \mathrm{MHz}$ are due to the interferometric conversion of the phase noise of the DFB laser into the intensity noise, and the spectrum around each frequency indicates the spectral profile of the DFB laser. If we 


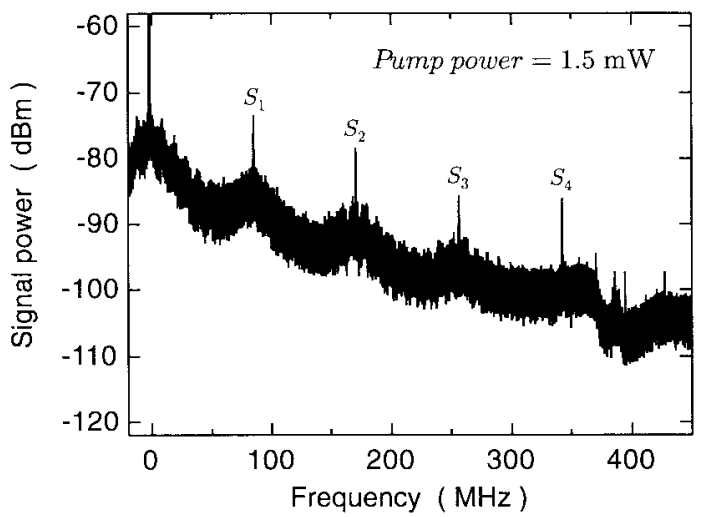

Fig. 3. An example of the measured multiplexed sensor signal spectrum.

use a low-coherence light source such as a superluminescent diode or a multilongitudinal mode semiconductor laser instead of the DFB laser, a flat noise floor can be achieved.

Fig. 4 shows the measured detailed signal spectrum of $S_{1} \sim$ $S_{4}$. The pump power to the EDFA was $1.5 \mathrm{~mW}$. The resolution of the spectrum analyzer was $1 \mathrm{kHz}$. In the experiment, $S_{1}$, $S_{2}, S_{3}$, and $S_{4}$ were sinusoidally phase-modulated at 5, 5.5, 6 , and $6.5 \mathrm{kHz}$, respectively. Good phase-modulated spectra are obtained.

Fig. 5 shows the sensitivities of $S_{1} \sim S_{4}$ against the pump power. The sensitivities are found to be enhanced by a factor of four with increasing the pump power.

\section{DISCUSSION}

In this sention, we theoretically analyze the output spectrum and the sensitivity of our sensor system. The configuration in Fig. 1 is again used for our analysis. For simplicity, we assume the following conditions:

1) the intensity fluctuation of the laser light is so small as to be neglected;

2) the phase fluctuation of the laser light is a Gaussian propability distribution;

3) the spectral profile of the laser light is Lorentzian;

4) the optical power from the laser is equally divided into all arms of the sensing interferometers.

The optical field amplitudes from the short and the long paths of the sensing interferometer $S_{m}(m=1,2, \cdots, n)$ at the input port of the optical loop, $E_{m S}$ and $E_{m L}$, respectively, are expressed as

$$
\begin{aligned}
& E_{m S}=\frac{\sqrt{P_{0}}}{4 n} \\
& E_{m L}=\frac{\sqrt{P_{0}}}{4 n} e^{-j \beta L_{m}}
\end{aligned}
$$

where $P_{0}$ is the incident optical power to the sensor system, $n$ is again the number of sensors to be multiplexed. $\beta$ is the propagation constant, and $L_{m}$ is again the OPD of $S_{m}$. Supposing the optical field with unit amplitude enters the input port of the fiber coupler (FC2). The amplitude of the beam passing directly through the loop, $e_{0}$, and the amplitude of the beam emerging from the loop after recirculating $k$-times in the loop, $e_{k}$, are writen as

$$
\begin{aligned}
& e_{0}=(1-\gamma)^{1 / 2}(1-\kappa)^{1 / 2} e^{j 2 \pi f_{0} t} \\
& e_{k}=\epsilon \eta^{k-1} e^{j 2 \theta} e^{-j k ; \beta} e^{j 2 \pi\left(f_{0}+k f_{F S}\right) t}
\end{aligned}
$$

where $\gamma$ and $\kappa$ are the excess loss and the coupling coefficient of the fiber coupler (FC2), respectively, $\theta(=\pi / 2)$ is the coupling phase shift of the fiber coupler, $f_{0}$ is the optical frequency of the emitted light from the light source, $T$ is the delay time of the optical loop, and,

$$
\begin{aligned}
& \epsilon=\kappa(1-\gamma)(1-\alpha)^{1 / 2} \\
& \eta=(1-\kappa)^{1 / 2}(1-\gamma)^{1 / 2}(1-\alpha)^{1 / 2}
\end{aligned}
$$

and $\alpha$ is the loop loss of the optical loop.

\section{A. Signal Power}

The sensor signal of $S_{k}$ is caused by the interference between the beam recirculating $i$-times in the loop after passing through a long path of $S_{k}$ and the beam recirculating $(i+k)$-times in the loop after passing through a short path of $S_{k}$. The sensor signal power of $S_{k}, S_{k}^{s i g}$, is calculated as

$$
\begin{aligned}
S_{k}^{s i g} & =\left|\sum_{i=0}^{\infty}\left(E_{k S S} e_{i+k}\right)\left(E_{k L} e_{i}\right)^{*}\right|^{2} \\
& =\frac{P_{0}^{2} \epsilon^{2}}{256 n^{4}} A^{2} \eta^{2(k-1)}
\end{aligned}
$$

where

$$
A=\frac{\epsilon \eta}{1-\eta^{2}}-\left|e_{0}\right|
$$

and $*$ indicates complex conjugate.

\section{B. Phase-Noise Induced Noise}

Since there are a large number of optical path differences which are longer than the coherence length of the light source in this system, the source phase-noise induced intensity noise is dominant in the noise spectrum and the shot noise and the thermal noise can be negligible. This can be confirmed by Fig. 4, which indicates that the noise spectrum consists of superposition of Lorentz distributions around $0, f_{F S}, 2 f_{F S}$, $3 f_{F S} \mathrm{~Hz}$, and so on. Therefore we first calculate the noise spectrum around each frequency and then add the resultant noise spectrum.

Let us first consider the noise spectrum around $0 \mathrm{~Hz}$. This noise is caused by the two beams recirculating the same times in the optical loop, and its intensity $I_{0}$ and the autocorrelation function of $I_{0}, R_{0}(\tau)$, are given as

$$
\begin{aligned}
I_{0} & =\left(\frac{\sqrt{P_{0}}}{4 n}\right)^{2} \sum_{i=0}^{\infty}\left|e_{i}\right|^{2} \\
& =\frac{P_{0}}{16 n^{2}}\left(\left|e_{0}\right|^{2}+\frac{\epsilon^{2}}{1-\eta^{2}}\right) \\
R_{0}(\tau) & =\left|I_{0}\right|^{2} e^{-2 \pi \Delta f|\tau|}
\end{aligned}
$$

where $\Delta f$ is the spectral linewidth of the light source. In (13), the term $\exp (-2 \pi \Delta f|\tau|)$ is caused by the phase fluctuation of the optical beam [8], [11], [12] with the assumptions described 


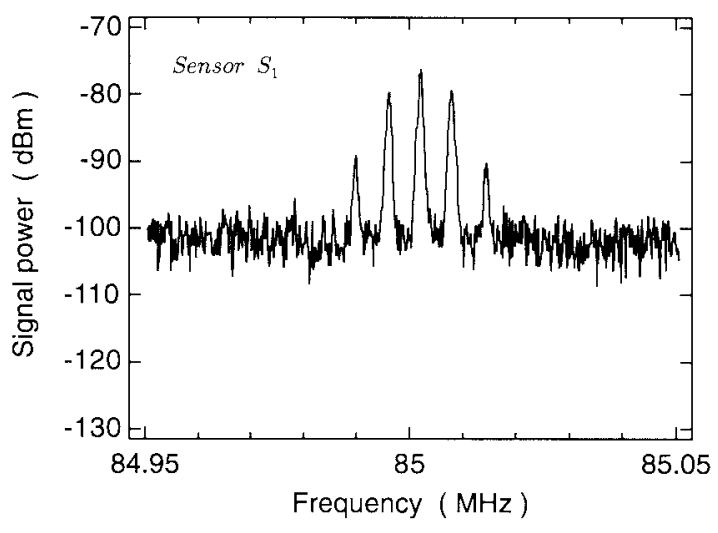

(a)

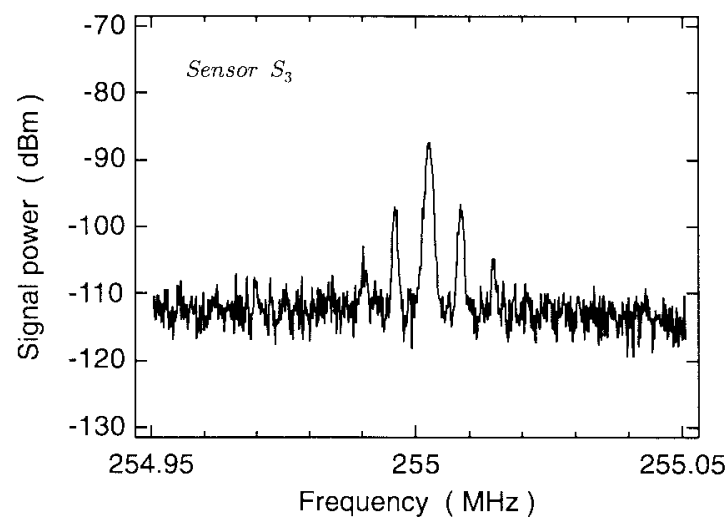

(c)

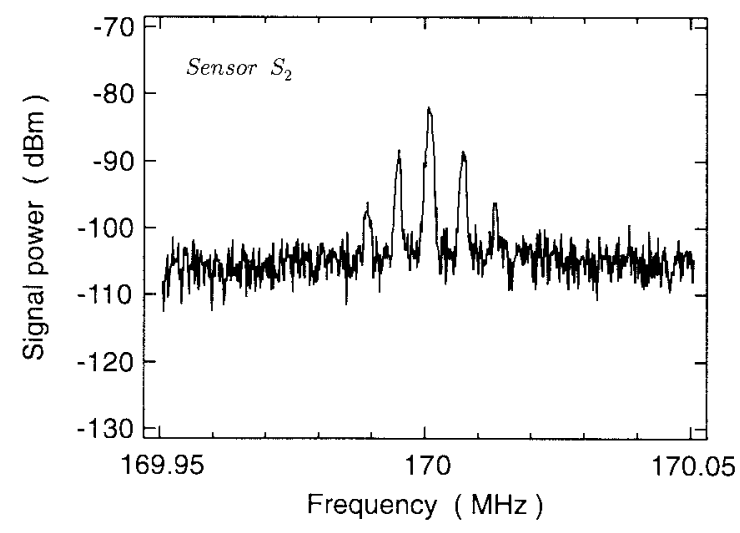

(b)

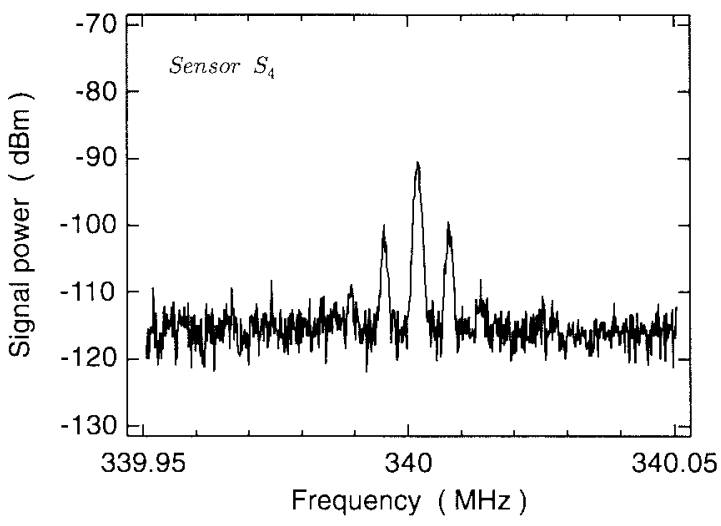

(d)

Fig. 4. Measured detailed signal spectrum of $S_{1} \sim S_{4}$. (a) Sensor $S_{1}$, (b) sensor $S_{2}$, (c) sensor $S_{3}$, and (d) sensor $S_{4}$.

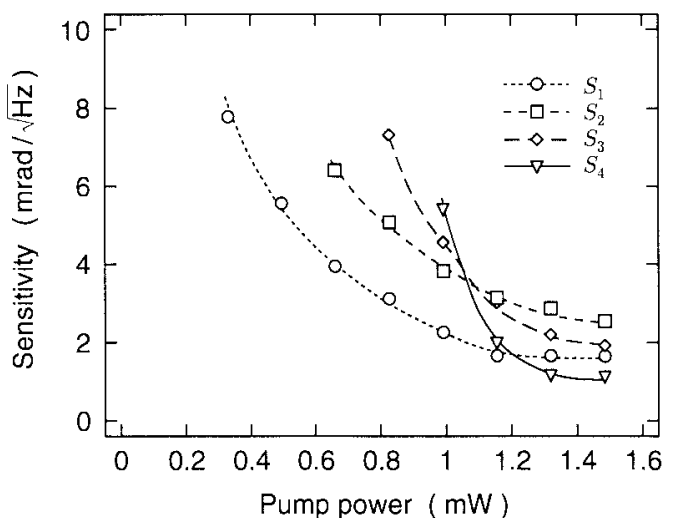

Fig. 5. Sensitivities of $S_{1} \sim S_{4}$ against the pump power.

above. The number of pairs of two beams contributing to the noise around $0 \mathrm{~Hz}$ is

$$
{ }_{2 n} C_{2}=n(2 n-1) \text {. }
$$

In this equation, $2 n$ is the number of the optical paths in the sensor array. Then the noise spectrum around $0 \mathrm{~Hz}, G_{0}(f)$, is given as

$$
\begin{aligned}
G_{0}(f) & =n(2 n-1) \mathcal{F}\left[R_{0}(\tau)\right] \\
& =n(2 n-1) \int_{-\infty}^{\infty} R_{0}(\tau) e^{-j 2 \pi f \tau} d \tau \\
& =\frac{P_{0}^{2}}{\pi} \frac{2 n-1}{256 n^{3}}\left(\left|e_{0}\right|^{2}+\frac{\epsilon^{2}}{1-\eta^{2}}\right)^{2} L(0, f)
\end{aligned}
$$

where $\mathcal{F}$ indicates the Fourier transform, and $L(0, f)$ is the Lorentz distribution expressed as

$$
L(0, f)=\frac{\Delta f}{\Delta f^{2}+f^{2}} .
$$

Next, we concern to the noise intensity at $\left(k \times f_{F S}\right) \mathrm{Hz}$ $(1 \leq k \leq n)$. This noise is caused by the two beams whose recirculation numbers in the loop are different by $k$-times. The intensity of the noise at $\left(k \times f_{F S}\right) \mathrm{Hz}, I_{k 1}$, and the autocorrelation function of $I_{k: 1}, R_{k 1}(\tau)$, are given by

$$
\begin{aligned}
I_{k 1} & =\left(\frac{\sqrt{P_{0}}}{4 n}\right)^{2} \sum_{i=0}^{\infty} e_{i}^{*} e_{i+k} \\
& =\frac{P_{0}}{16 n^{2}} \epsilon \eta^{k-1} e^{j 2 \pi k f_{F S} t}\left(\frac{\epsilon \eta}{1-\eta^{2}}-\left|e_{0}\right|\right) \\
R_{k 11}(\tau) & =\left|I_{k 1}\right|^{2} e^{-2 \pi \Delta f|\tau|} e^{j 2 \pi k f_{F S} \tau} .
\end{aligned}
$$

The number of pairs of two beams appearing at $\left(k \times f_{F S}\right) \mathrm{Hz}$ is

$$
{ }_{2 n} P_{2}+2 n=4 n^{2}
$$

and only a pair of the two beams contributes to the signal and $\left(4 n^{2}-1\right)$ pairs of the two beams contribute to the noise. Then the noise spectrum around $\left(k \times f_{F S}\right) \mathrm{Hz}, G_{k 1}(f)$, is given as

$$
\begin{aligned}
G_{k 11}(f) & =\left(4 n^{2}-1\right) \mathcal{F}\left[R_{k: 1}(\tau)\right] \\
& =\left(4 n^{2}-1\right) \int_{-\infty}^{\infty} R_{k 11}(\tau) e^{-j 2 \pi f \tau} d \tau \\
& =\frac{P_{0}^{2}}{\pi} \frac{4 n^{2}-1}{256 n^{4}} A^{2} L(k, f) \eta^{2(k-1)}
\end{aligned}
$$


where $L(k, f)$ is the Lorentz distribution expressed as

$$
L(k, f)=\frac{\Delta f}{\Delta f^{2}+\left(f-k \times f_{F S}\right)^{2}} .
$$

Finally, we calculate the noise intensity at $\left(k \times f_{F S}\right) \mathrm{Hz}$ $(k \geq n+1)$. The number of pairs of two beams appearing at $\left(k \times f_{F S}\right) \mathrm{Hz}$ is

$$
{ }_{2 n} P_{2}+2 n=4 n^{2}
$$

and all the pairs contribute to noise. The noise intensity $I_{k: 2}$ and the autocorrelation function of $I_{k 2}, R_{k 2}(\tau)$, are calculated as

$$
\begin{aligned}
I_{k 2} & =\left(\frac{\sqrt{P_{0}}}{4 n}\right)^{2} \sum_{i=0}^{\infty} e_{i}^{*} e_{i+k} \\
& =\frac{P_{0}}{16 n^{2}} \epsilon \eta^{k-1} e^{j 2 \pi k f_{F S} t}\left(\frac{\epsilon \eta}{1-\eta^{2}}-\left|e_{0}\right|\right) \\
R_{k 2}(\tau) & =\left|I_{k 2}\right|^{2} e^{-2 \pi \Delta f|\tau|} e^{j 2 \pi k f_{F S} \tau} .
\end{aligned}
$$

Then the noise spectrum around $\left(k \times f_{F S}\right) \mathrm{Hz}, G_{k 2}(f)$, is given as

$$
\begin{aligned}
G_{k 2}(f) & =4 n^{2} \mathcal{F}\left[R_{k 2}(\tau)\right] \\
& =4 n^{2} \int_{-\infty}^{\infty} R_{k 2}(\tau) e^{-j 2 \pi f \tau} d \tau \\
& =\frac{P_{0}^{2}}{\pi} \frac{A^{2}}{64 n^{2}} L(k, f) \eta^{2(k-1)} .
\end{aligned}
$$

Thus, the noise spectrum $G(f)$ is obtained as

$$
G(f)=G_{0}(f)+\sum_{k=1}^{n} G_{k 1}(f)+\sum_{k=n+1}^{\infty} G_{k 2}(f) .
$$

Hence, the output spectrum $S(f)$ is given by

$$
\begin{aligned}
S(f) & =\sum_{k=1}^{n} S_{k}^{s i g}+B_{W} G(f) \\
& =\frac{P_{0}^{2} \epsilon^{2}}{256 n^{4}} A^{2} \sum_{k=1}^{n} \eta^{2(k-1)} \delta\left(f-k f_{F S}\right) \\
& +\frac{B_{W} P_{0}^{2}}{\pi} \frac{2 n-1}{256 n^{3}}\left(\left|e_{0}\right|^{2}+\frac{\epsilon^{2}}{1-\eta^{2}}\right)^{2} L(0, f) \\
& +\frac{B_{W} P_{0}^{2} \epsilon^{2}}{\pi} \frac{4 n^{2}-1}{256 n^{4}} A^{2} \sum_{k=1}^{n} L(k, f) \eta^{2(k-1)} \\
& +\frac{B_{W} P_{0}^{2} \epsilon^{2}}{\pi} \frac{A^{2}}{64 n^{2}} \sum_{k=n+1}^{\infty} L(k, f) \eta^{2(k-1)}
\end{aligned}
$$

where $B_{W}$ is the bandwidth of the detecting apparatus (for example, the spectrum analyzer). In (27), the first term is the signal intensity and the second to fourth terms are the noise profiles.

When a sinusoidal phase shift is applied to $S_{k}$, the sensor signal $P_{k}$ can be expressed as

$$
\begin{aligned}
P_{k} & \propto \cos \left\{2 \pi k f_{F S} t+\phi_{k} \sin \left(2 \pi f_{k} t\right)\right\} \\
& =\sum_{i=-\infty}^{\infty} J_{i}\left(\phi_{k}\right) \cos \left\{2 \pi\left(k f_{F S}+i f_{k}\right) t+\frac{n \pi}{2}\right\}
\end{aligned}
$$

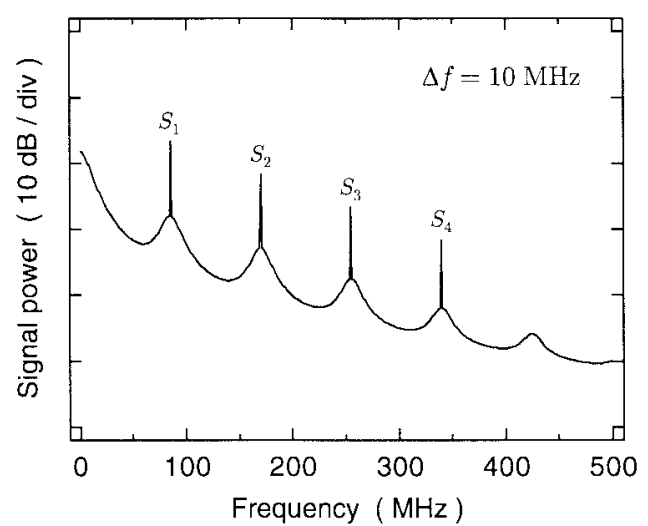

(a)

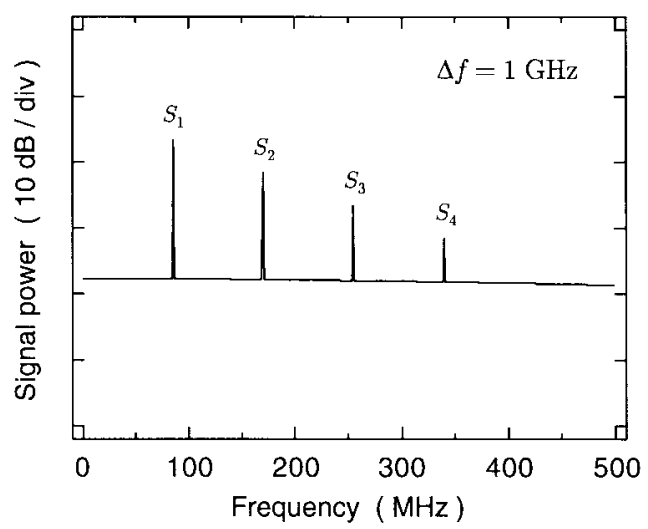

(b)

Fig. 6. Calculated sensor signal spectrum for four-sensors $\left(S-1 \sim S_{4}\right)$ multiplexed system. (a) $\Delta f=10 \mathrm{MHz}$ and (b) $\Delta f=1 \mathrm{GHz}$.

where $\phi_{k}$ is the phase amplitude of the phase shift and $f_{k}$ is the frequency of the phase shift. The phase shift can be measured from the magnitude of the first sideband around $\left(k \times f_{F S}\right) \mathrm{Hz}$, which is given as $J_{1}\left(\phi_{k}\right)$. If $\phi_{k} \ll 1, J_{1}\left(\phi_{k}\right) \approx \phi_{k} / 2$, and the squared sensor signal is

$$
S_{k}^{s i g}=\left(\frac{P_{0}}{16 n^{2}}\right)^{2} \epsilon^{2} A^{2} \eta^{2(k-1)} \frac{\phi_{k}^{2}}{4} .
$$

By using (29), the sensitivity of $S_{k}, \phi_{k, m i n}$, is given as

$$
\phi_{k, \text { min }}=\frac{2}{\sqrt{B_{W}}} \sqrt{\frac{S_{k}^{s i g}}{G\left(k \times f_{F S}\right)}} \quad(\mathrm{rad} / \sqrt{\mathrm{Hz}}) .
$$

Fig. 6 shows the calculated spectrum for four-sensors $\left(S_{1} \sim\right.$ $S_{4}$ ) multiplexed system for (a) $\Delta f=10 \mathrm{MHz}$ and (b) $\Delta f=1$ GHz. In the calculation, $\alpha=0.5, \gamma=0.2, \kappa=0.5$, $B_{W}=100 \mathrm{kHz}$ were used, which are the same condition with the experiment of Fig. 3. The calculated result shown in Fig. 6(a) agrees well with the experimental result shown in Fig. 3. This result confirms that the noise spectrum in this sensor system is mainly dominated by the source phase-noise induced intensity noise. When $\Delta f=1 \mathrm{GHz}$, the noise level is found to be almost constant.

Fig. 7 shows the calculated sensitivities for $S_{1} \sim S_{4}$ against $\kappa$ for (a) $\Delta f=10 \mathrm{MHz}$ and (b) $\Delta f=1 \mathrm{GHz}$. When $\Delta f=10$ $\mathrm{MHz}$, the sensitivities for $S_{1} \sim S_{4}$ are almost the same value when $\kappa$ ranges from 0.2 to 0.6 because the noise level as well 


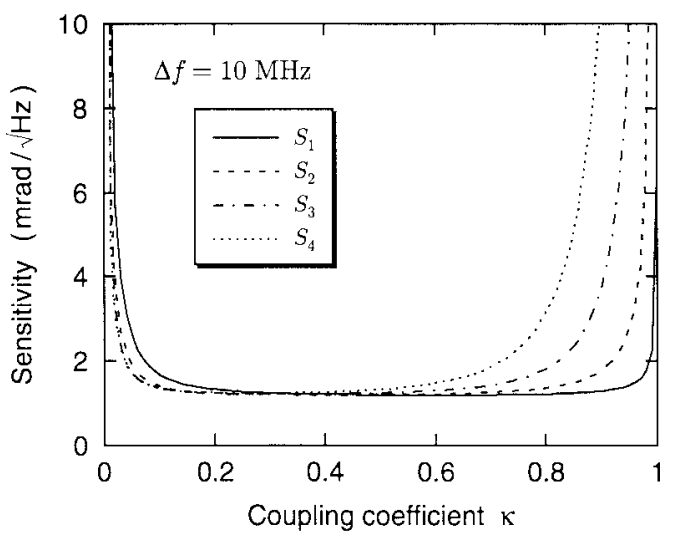

(a)

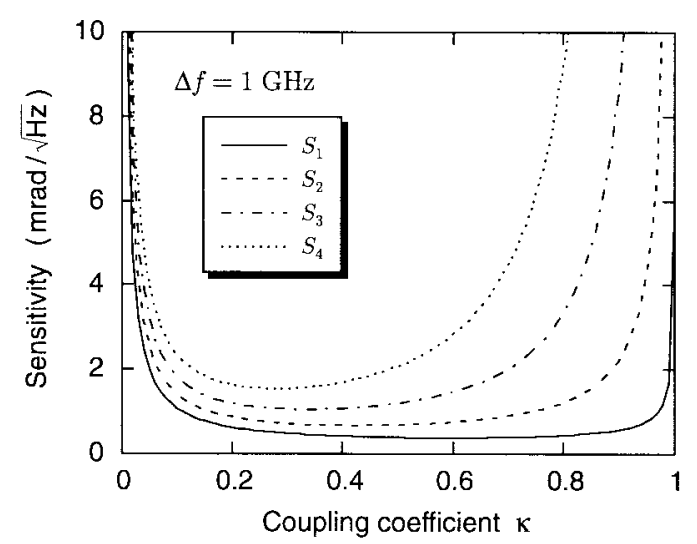

(b)

Fig. 7. Calculated sensitivities for $S_{1} \sim S_{4}$ against $\kappa$. (a) $\Delta f=10 \mathrm{MHz}$ and (b) $\Delta f=1 \mathrm{GHz}$.

as the signal power decreases with increasing the frequency as shown in Figs. 3 and 6, and the sensitivities in this range of $\kappa$ are minimum. The value of $\kappa$ in this range is, therefore, the optimum value. On the other hand, the sensitivity for the larger sensor number decreases when $\Delta f=1 \mathrm{GHz}$. This is because the signal power decreases for the larger sensor number while the noise level is almost constant as shown in Fig. 6(b). The optimum value of $\kappa$ in this case is between 0.2 and 0.4 . The sensitivities can be equalized by optimizing the power splitting ratio of the tree coupler shown in Fig. 1.

Fig. 8 shows the calculated sensitivities for $S_{1} \sim S_{4}$ against $\alpha$ for (a) $\Delta f=10 \mathrm{MHz}$ and (b) $\Delta f=1 \mathrm{GHz}$. When $\Delta f=10$ $\mathrm{MHz}$, the sensitivities for $S_{1} \sim S_{4}$ are almost same when $\alpha$ ranges from 0 to 0.4 because of the same reason for Fig. 7(a), and the sensitivities in this range of $\alpha$ are minimum. The value of $\alpha$ in this range is, therefore, the optimum value. On the other hand, the sensitivity for the larger sensor number decreases when $\Delta f=1 \mathrm{GHz}$ because of the same reason for Fig. 7(b). The optimum value of $\alpha$ in this case is about 0.2 .

Fig. 9 shows the calculated sensitivities against the sensor number for ten sensors multiplexed system $(n=10)$. When the spectral linewidth $\Delta f$ is small, the sensitivities for all sensors are almost same at the sacrifice of the sensitivities for the sensors with small number. It is found from this figure that the light source with narrow spectral linewidth is preferable when many sensors are multiplexed, and the light source with

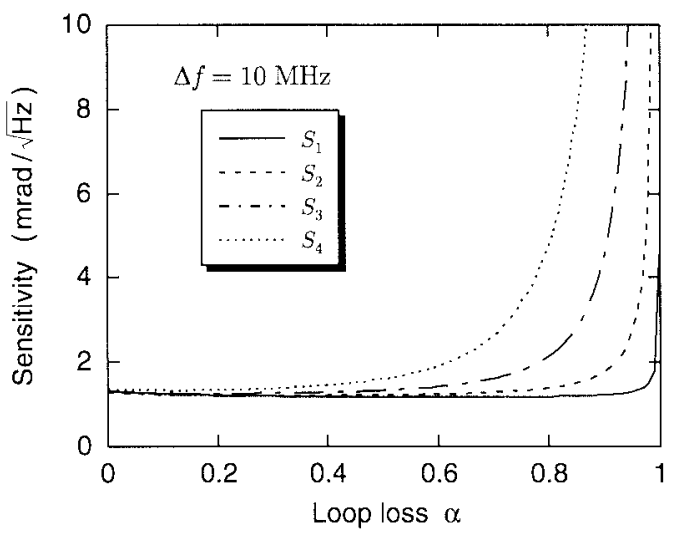

(a)

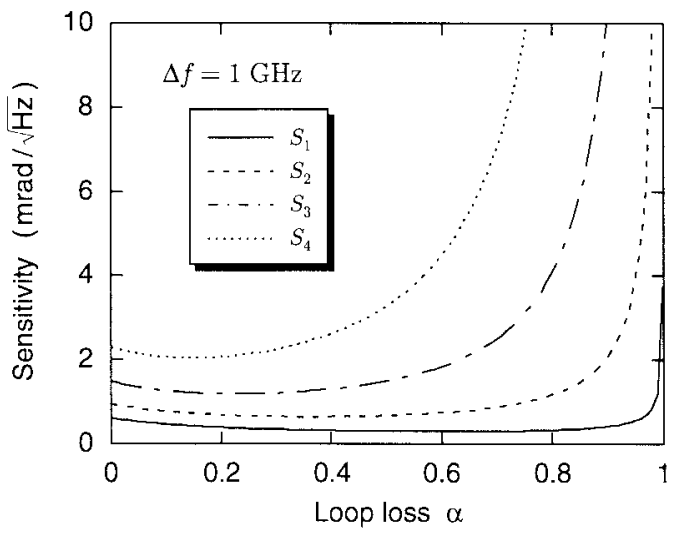

(b)

Fig. 8. Calculated sensitivities for $S_{1} \sim S_{4}$ against $\alpha$. (a) $\Delta f=10 \mathrm{MHz}$ and (b) $\Delta f=1 \mathrm{GHz}$.

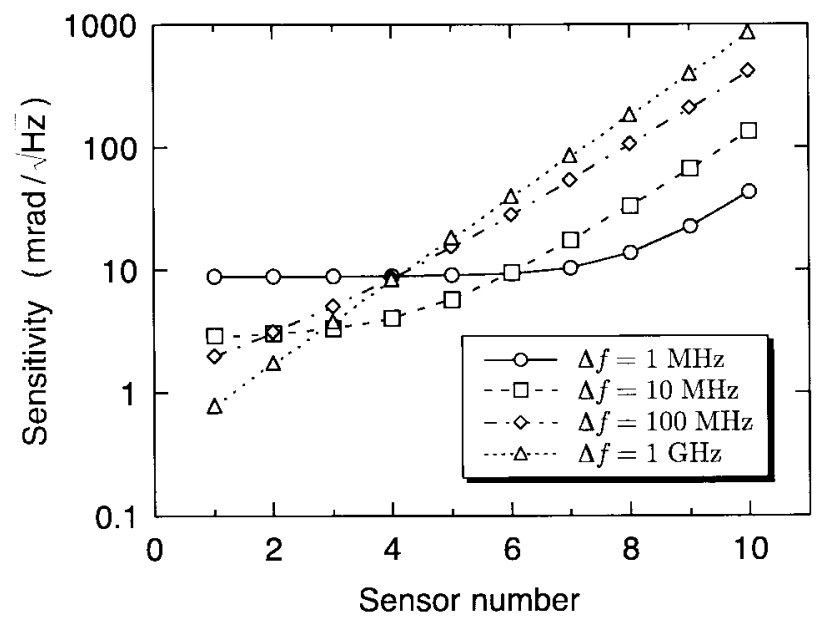

Fig. 9. Calculated sensitivities against the sensor number for ten sensors multiplexed system.

broad spectral linewidth is preferable when a few sensors are multiplexed.

\section{CONCLUSION}

We have demonstrated a frequency domain detection scheme of the coherence multiplexed sensor signals by using an optical loop with a frequency shifter. In this method, by 
adjusting the optical path differences of the sensors to integermultiple of the loop length of the optical loop, the sensor signals are multiplexed in the frequency domain, appearing at the integer-multiple of the working frequency of the frequency shifter, and consequently, the numbers of the receiving interferometers and the photodetectors can be reduced. In the experiments, four sensors are successfully multiplexed by inserting an erbium-doped optical fiber amplifier in the optical loop to reduce the loop loss, and the sensitivities of about 2 $\mathrm{mrad} / \sqrt{\mathrm{Hz}}$ are obtained.

Next, we theoretically discuss the sensitivities by considering the source phase-noise induced intensity noise. The calculated spectrum and the sensitivities are in good agreement with the experimental results. The influence of the loop loss $\alpha$, the coupling coefficient of the fiber coupler, $\kappa$, and the spectral linewidth of the light source $\Delta f$, are also theoretically investigated.

\section{REFERENCES}

[1] J. L. Brooks, M. Tur, B. Y. Kim, K. A. Fesler, and H. J. Shaw, "Fiber-optic interferometric sensor arrays with freedom from source phase-induced noise," Opt. Lett., vol. 11, no. 7, pp. 473-475, 1986.

[2] A. D. Kersey, K. L. Dorsey, and A. Dandridge, "Demonstration of an eight-element time-division multiplexed interferometric fiber sensor array," Electron. Lett., vol. 24, no. 11, pp. 689-691, 1988.

[3] I. Sakai, G. Parry, and R. C. Youngquist, "Multiplexing fiber-optic sensors by frequency modulation: Cross-talk consideration," Opt. Lett., vol. 11 , no. 3 , pp. $183-185,1986$

[4] D. T. Jong and K. Hotate, "Frequency division multiplexing of optical fiber sensors using an optical delay loop with a frequency shifter," Appl. Opt., vol. 28, no. 7, pp. 1289-1297, 1989.

[5] J. L. Santos, F. Farahi, T. Newson, A. P. Leite, and D. A. Jackson: "Frequency multiplexing of remote all-fiber Michelson interferometers with lead insensitivity," J. Lightwave Technol., vol. 10, no. 6, pp. 853-863, 1992.

[6] J. L. Brooks., R. H. Wentworth, R. C. Youngquist, M. Tur, B. Y. Kim, and H. J. Shaw, "Coherence multiplexing of fiber-optic interferometric sensors," J. Lightwave Technol., vol. LT-3, pp. 1062-1072, 1985.

[7] A. D. Kersey and A. Dandridge: "Phase-noise reduction in coherencemultiplexed interferometric fiber sensors," Electron. Lett., vol. 22, no.
11, pp. 616-617, 1986.

[8] R. H. Wentworth, "Theoretical noise performance of coherencemultiplexed interferometric sensors," J. Lightwave Technol., vol. 7, pp. 941-956, 1989.

[9] K. Iiyama, M. Harano, K. Hayashi, Y. Ida, T. Kadoshima, and Y. Kurasawa, "Detection scheme of coherence multiplexed sensor signals by using optical loop incorporating frequency shifter," Electron. Lett., vol. 28 , no. 2 , pp. $169-170,1992$.

[10] X. Zhou, K. Iiyama, and K. Hayashi, "Detection scheme of coherence multiplexed sensor signals by using an optical loop with a frequency shifter: Sensitivity enhancement," IEEE Photon. Technol. Lett., vol. 6, pp. 767-769, June 1994.

[11] M. Tur, B. Moslehi, and J. W. Goodman, "Theory of laser phase noise in recirculating fiber-optic delay line," J. Lightwave Technol., vol. LT-3, pp. 20-31, Jan. 1985.

[12] R. W. Tkach and A. R. Chraplyvy, "Phase noise and linewidth in an InGaAsP DFB laser," J. Lightwave Technol., vol. LT-4, pp. 1711-1716, Nov. 1996.

Koichi Iiyama (M'95) was born in Fukui, Japan, on March 19, 1963. He received the B.E., M.E., and D.E. degrees in electronics all from Kanazawa University, Japan, in 1985, 1987, and 1993, respectively.

From 1987 to 1988, he worked at Yokogawa Hewlett-Packard, Ltd. Since 1988, he has been working in the Department of Electrical and Computer Engineering, Faculty of Engineering, Kanazawa University, and is now an Associate Professor. He is currently working in research on optical reflectometry, fiber-optic sensors, optical fiber amplifiers, and optical waveguide devices.

Dr. Iiyama is a member of the Institute of Electronics, Information and Communication Engineers (IEICE) and the Japan Society of Applied Physics.

Ken-ichi Hayashi (M'88) was born in Kanazawa, Japan, on July 14, 1943. He died of illness in May 1996. He had received the B.E. degree in electronics from Kanazawa University, Japan, in 1966, and the D.E. degrees from Nagoya University, Japan, in 1977.

From 1966 to 1996, he had been with the Department of Electronics, Kanazawa University. He had been engaged in research on optical reflectometry, fiber-optic sensors, optical fiber amplifiers, and optical waveguide devices. 